\title{
Miocarditis por Sars-Cov-2, en paciente con hisopado nasofaríngeo negativo. Primer caso descrito en Costa Rica
}

\author{
Dr. José Alejandro Sanabria-Garita ${ }^{1}$ \\ Dr. Carlos Eduardo Bolaños-Gómez ${ }^{2}$ \\ Dr. Juan Carlos Vega-Chaves ${ }^{2}$ \\ Dr. Edgar Fuentes-Molina ${ }^{3}$ \\ Dr. Alejandro Vallecillo-Torres ${ }^{4}$
}

\section{Correspondencia}

Carlos Eduardo Bolaños-Gómez

carlosbolanos.gomez@gmail.com

${ }^{1}$ Cardiólogo Clínico, profesor Postgrado Cardiología Universidad de Costa Rica, Coordinador Unidad Cuidados Cardiacos Agudos, Hospital San Juan de Dios. ${ }^{2}$ Residentes Posgrado en Cardiología, Universidad de Costa Rica.

${ }^{3}$ Cardiólogo Clínico, profesor Postgrado Cardiología Universidad de Costa Rica, Coordinador Laboratorio de Ecocardiografía Hospital San Juan de Dios, Máster en Imagen Cardiaca.

${ }^{4}$ Especialista en Radiología, Servicio de Radiología, Hospital San Juan de Dios, Máster en Resonancia Magnética Cardiaca.

Enviado: 13/03/2021.

Aceptado: 4/11/2021.

Publicado: 15/12/2021

Citar como: Sanabria-Garita JA, Bolaños-Gómez CE, Vega-Chaves JC, Fuentes-Molina E, Vallecillo-Torres E: Miocarditis por Sars-Cov-2, en paciente con hisopado nasofaríngeo negativo. Primer caso descrito en Costa Rica. RETIC. 2021 (Diciembre); 4 (3): 39-43. doi: 10.37615/retic.v4n3a11

Cite this as: Sanabria-Garita JA, Bolaños-Gómez CE, Vega-Chaves JC, Fuentes-Molina E, Vallecillo-Torres E: Sars-Cov-2 myocarditis in a patient with a negative nasopharyngeal swab. First case described in Costa Rica. RETIC. 2021 (December); 4 (3): 39-43. doi: 10.37615/retic.v4n3a11

\section{Palabras clave \\ $\triangleright$ Lesión miocárdica. \\ $\triangleright$ Miocarditis \\ $\triangleright$ COVID 19 .}

\section{RESUMEN}

La miocarditis asociada a la enfermedad por SARS-CoV-2, es conocida y se han notificado varios casos a nivel mundial. Se cree que la fisiopatología de la miocarditis relacionada con COVID-19 es una combinación del daño viral directo al miocardio y daño cardíaco debido a la respuesta inmune del huésped. Los hallazgos clínicos incluyen cambios en el electrocardiograma, alteración de los biomarcadores cardiacos y deterioro de la función cardiaca. Dentro del trabajo diagnóstico se debe descartar la enfermedad arterial coronaria, y hacer uso de técnicas de imagen cardiaca como la resonancia magnética cardiaca, los cuales permiten identificar los criterios de Lake Louis, característicos de la miocarditis.

ABSTRACT
Myocarditis associated with SARS-CoV-2 disease is known and several cases have been reported worldwide. The
pathophysiology of COVID-19-related myocarditis is believed to be a combination of direct viral damage to the
myocardium and cardiac damage due to the host's immune response. Clinical findings include changes in the
electrocardiogram, alteration of cardiac biomarkers, and cardiac function impairment. Within the diagnostic
work, coronary artery disease must be ruled out, and cardiac imaging techniques such as cardiac magnetic reso-
nance should be used, allowing the Lake Louis criteria, characteristic of myocarditis, to be identified.

\section{Presentación de Caso Clínico}

Hombre de 51 años, hipertenso, diabético, dislipidémico y tabaquista activo; con antecedente de infección leve por SARS-CoV-2 (diagnosticado por nexo epidemiológico) desde hacía dos semanas. Consulta al servicio de emergencias por cuadro de varios días de disnea de moderados esfuerzos, disnea paroxística nocturna, ortopnea, náuseas y fatiga de varios días de evolución.

Al examen físico se encontraba taquipneico, con ingurgitación yugular y reflujo hepatoyugular a 45 grados, con crépitos gruesos húmedos difusos, sin soplos a la auscultación.
Se le realizó prueba PCR para SARS-Cov-2 mediante hisopado nasofaríngeo, que resultó negativa. Laboratorios generales documentaron: leucocitosis con neutrofilia y linfopenia, anemia normocítica-normocrómica, azoemia prerrenal, patrón colestásico, Pro-BNP 21.165 pg/ml y troponina T 59 ng/L. Adicionalmente, se solicitó cuantificación de anticuerpos contra SARS-CoV-2, documentando presencia de inmunoglobulinas M y G (IgM e lgG), que indicaba una infección reciente, en fase de resolución.

La radiografía de tórax evidenció infiltrados algodonosos bilaterales, con cefalización de flujo. El electrocardiograma presentó ritmo sinusal con elevación del ST de V1-V3, y aplanamiento de las ondas T en todas las derivaciones (Figura 1). 


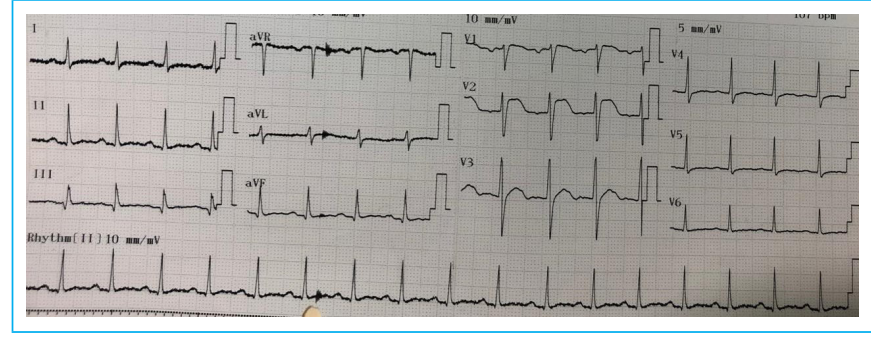

Figura 1. Electrocardiograma de 12 derivaciones.

Se practicó ecocardiograma transtorácico (ETT) basal el cual reportó hipocinesia difusa predominantemente de los segmentos medios y apicales del ventrículo izquierdo (VI), con fracción de eyección automática de 39\%, insuficiencia mitral leve, insuficiencia tricuspídea moderada, dilatación de ambas aurículas, baja probabilidad de hipertensión pulmonar y ausencia de derrame pericárdiCo, con strain longitudinal global del VI de $-10.9 \%$ (videos 1 y 2 , figuras 2 y 3 ).

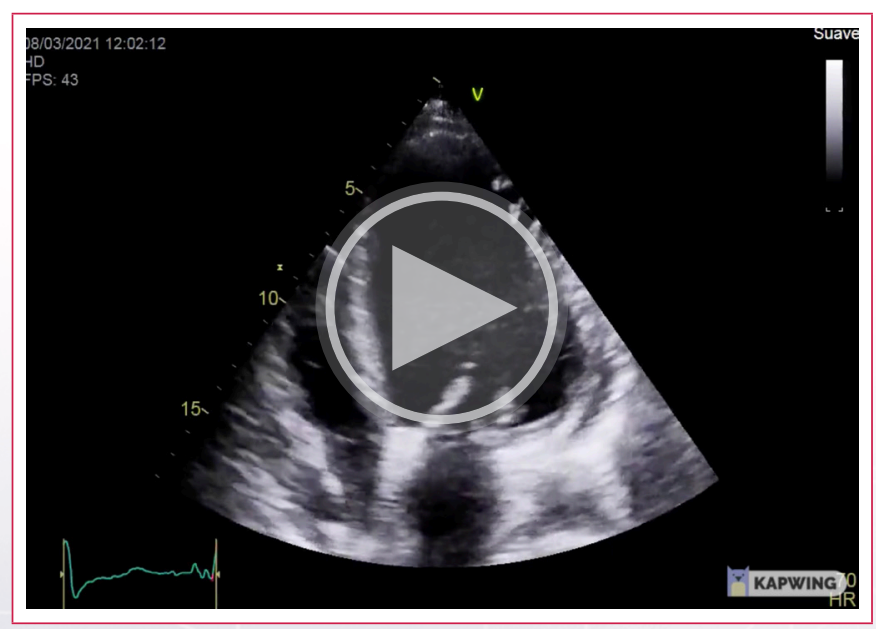

Vídeo 1. Ecocardiograma transtorácico basal, tomas 4 y 2 cámaras apical, que muestra reducción de fracción de eyección al ingreso a la unidad de cuidados coronarios.

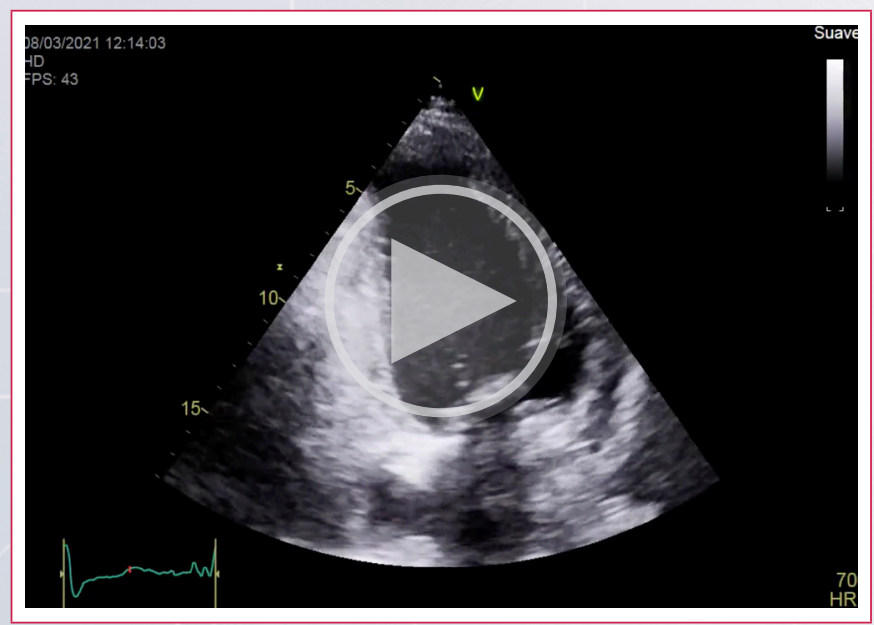

Vídeo 2. Ecocardiograma transtorácico basal, tomas 4 y 2 cámaras apical, que muestra reducción de fracción de eyección al ingreso a la unidad de cuidados coronarios.

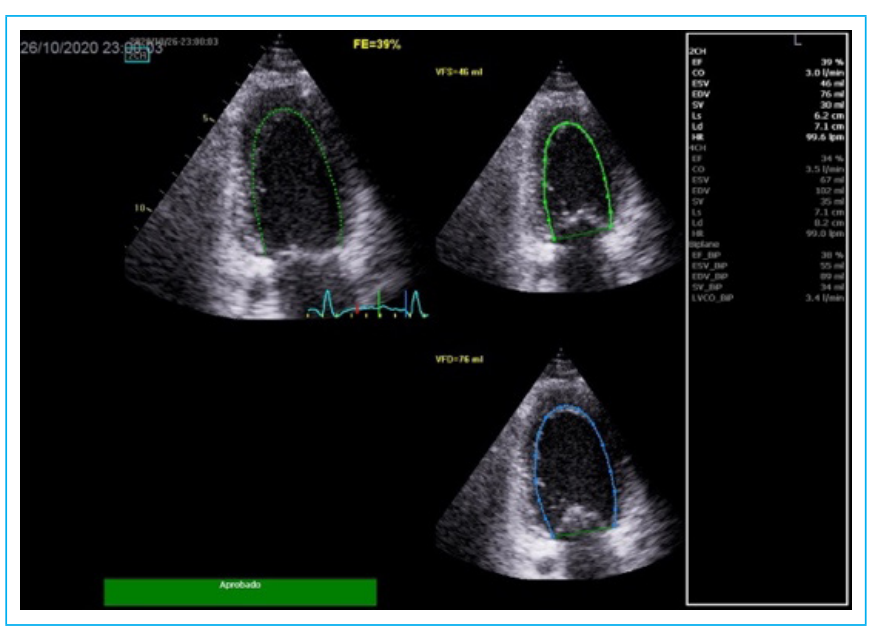

Figura 2. Ecocardiograma transtorácico basal que muestra reducción de fracción de eyección (automática) al ingreso a la unidad de cuidados coronarios.
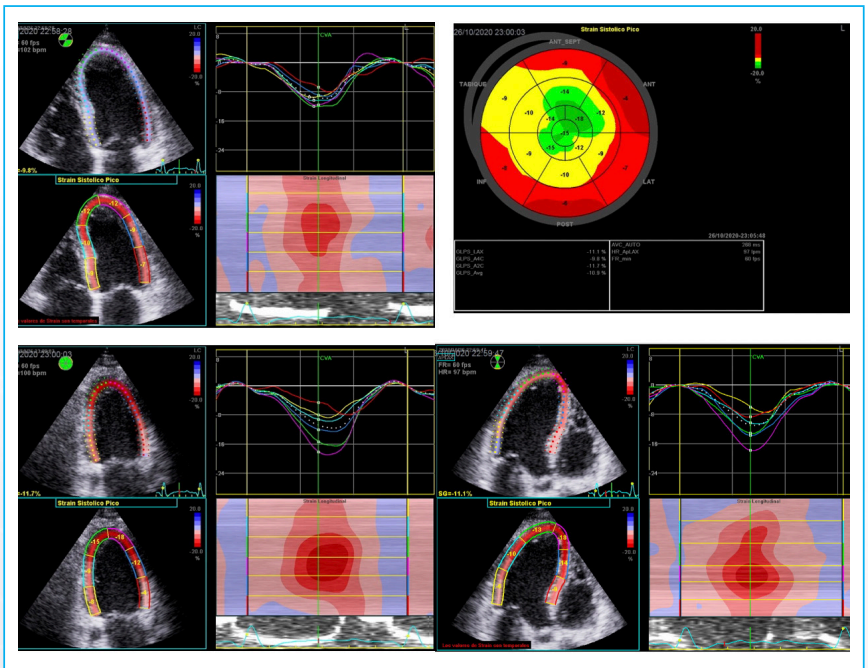

Figura 3. Strain longitudinal global al ingreso a la unidad de cuidados coronario.

Se inició terapia con diuréticos a altas dosis, solución hipertónica al 1.5\% y oxígeno suplementario por cánula nasal a $4 \mathrm{~L} / \mathrm{min}$. El paciente mejoró y se logró una reducción de más de un 50\% del Pro-BNP con respecto al de ingreso (control: $8.000 \mathrm{pg} / \mathrm{mL}$ ).

Nuestro desafío fue definir si las alteraciones a nivel miocárdico eran agudas o crónicas descompensadas, por lo que se completó la evaluación con otros métodos complementarios.

Se practicó angiografía coronaria que no reportó lesiones o placas significativas.

Se complementó el estudio de imagen cardiaca con una resonancia magnética que evidenció datos sugestivos de edema miocárdico difuso (figuras 4 y 5) y ausencia de realce tardío miocárdico luego de inyección de Gadolinio indicando ausencia de fibrosis focal (Figuras 6 y 7 ). 


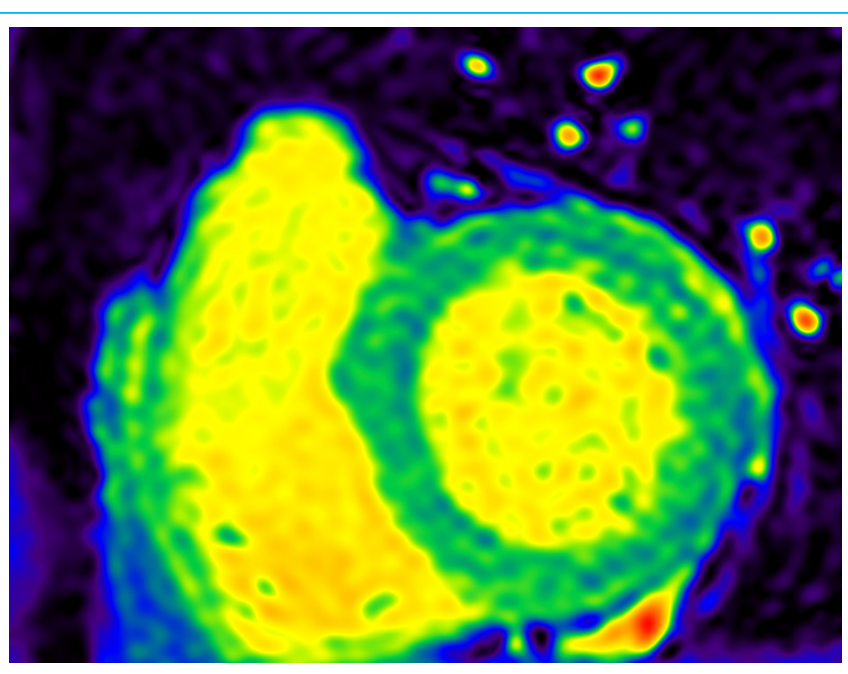

Figura 4. Resonancia magnética cardiaca. "T1 Mapping" nativo, secuencia MOLLI a 1.5 T, eje corto medio-ventricular izquierdo; se documentan valores superiores a 1000 ms, sugestivos de edema miocárdico difuso.

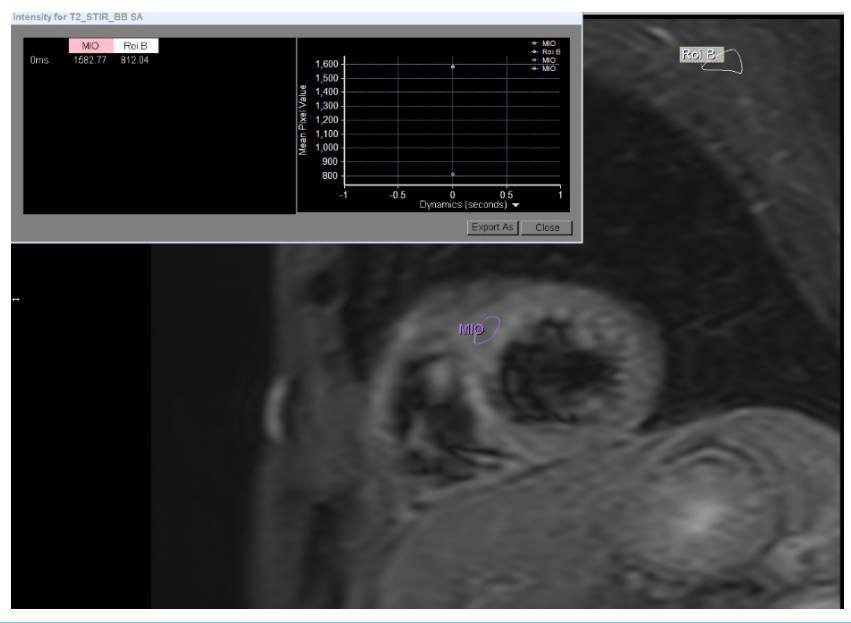

Figura 5. Resonancia magnética cardiaca. Secuencia T2-STIR, eje corto medioventricular izquierdo; relación de intensidad miocardio/músculo esquelético: 1.94, congruente con edema miocárdico.

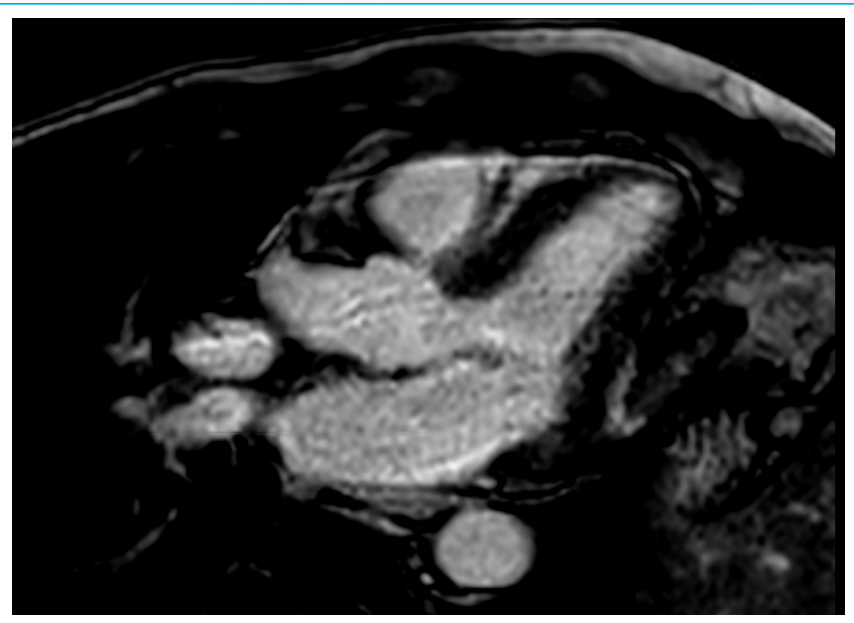

Figura 6. Resonancia magnética cardiaca. Secuencia IRTFE, eje largo, 10 minutos posterior a la administración de gadolinio: ausencia de realce tardío miocárdico, congruente con ausencia de necrosis significativa y/ o fibrosis precoz.

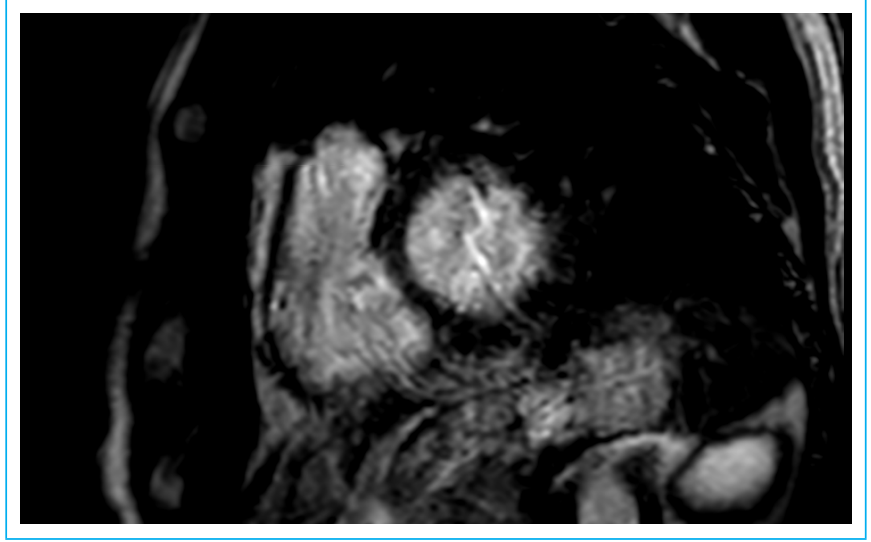

Figura 7. Resonancia magnética cardiaca. Secuencia IRTFE, eje corto del ventrículo izquierdo, 10 minutos posterior a la administración de gadolinio: ausencia de realce tardío miocárdico, congruente con ausencia de necrosis significativa y/o fibrosis precoz.

El paciente tuvo buena evolución clínica, se instauró tratamiento con Enalapril (IECA) y Carvedilol y se inició manejo ambulatorio tras 5 días de hospitalización.

A los tres meses, se practicó ETT control que mostró recuperación de la contractilidad del ventrículo izquierdo, con mejoría de la fracción de eyección del VI a 51\%, y del strain longitudinal global a -19.6\%, concordante con una Miocarditis en fase de recuperación, tras la infección por SARS- CoV-2 (videos 3 y 4, figuras 8 y 9).

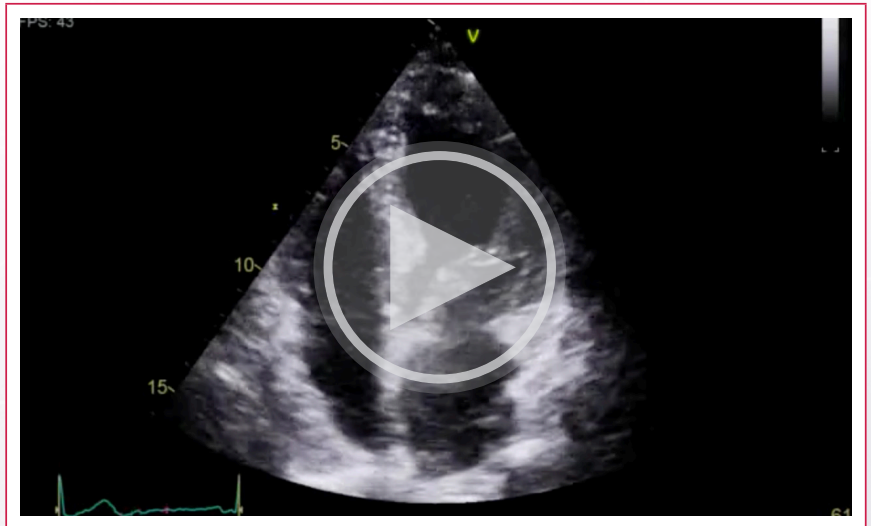

Vídeo 3. Ecocardiograma transtorácico a los 3 meses de tratamiento anti falla cardíaca, tomas 4 y 2 cámaras apical, que muestran recuperación de la fracción de eyección

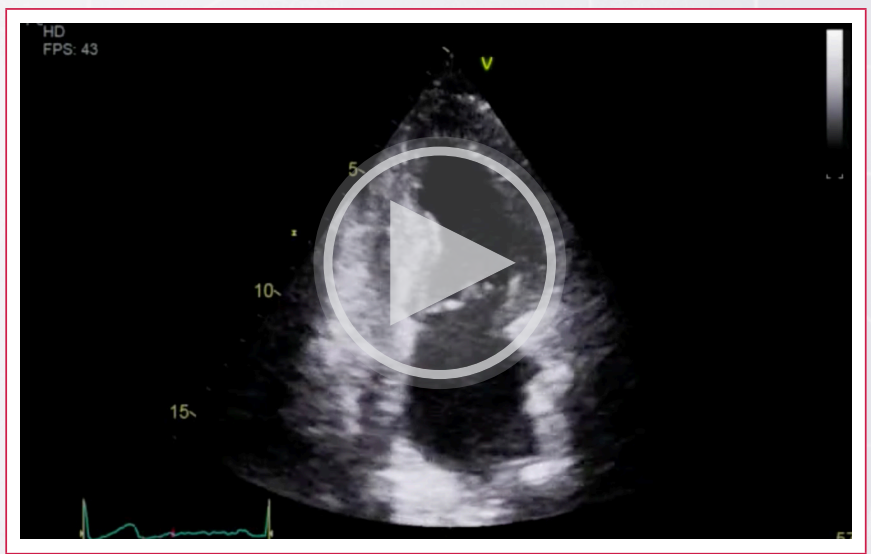

Vídeo 4. Ecocardiograma transtorácico a los 3 meses de tratamiento ant falla cardíaca, tomas 4 y 2 cámaras apical, que muestran recuperación de la fracción de eyección. 


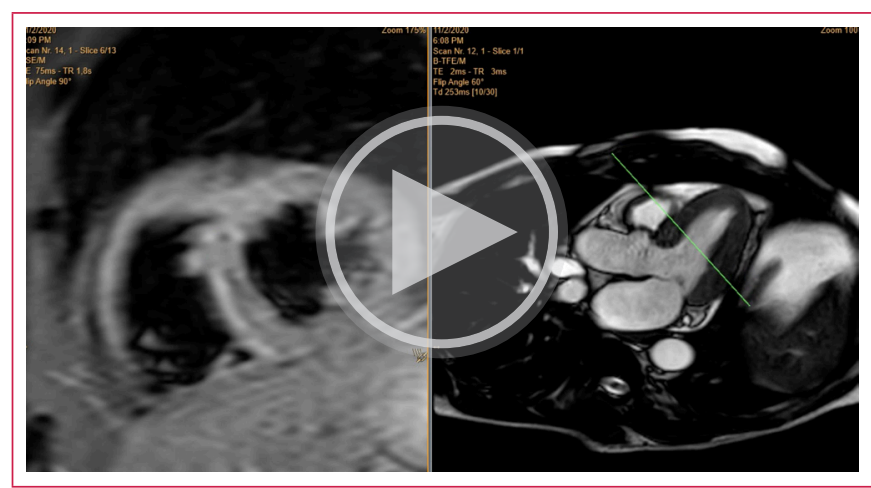

Vídeo 5. Resonancia magnética cardiaca. Secuencia T2-STIR, secuencia estática base-ápex (izquierda) y secuencia cine ("white blood") del eje largo del ventrículo izquierdo (derecha); se observan zonas de hiperintensidad T2 en los segmentos apicales, septales y anteriores (predominio en segmentos medios), congruentes con edema miocárdico.

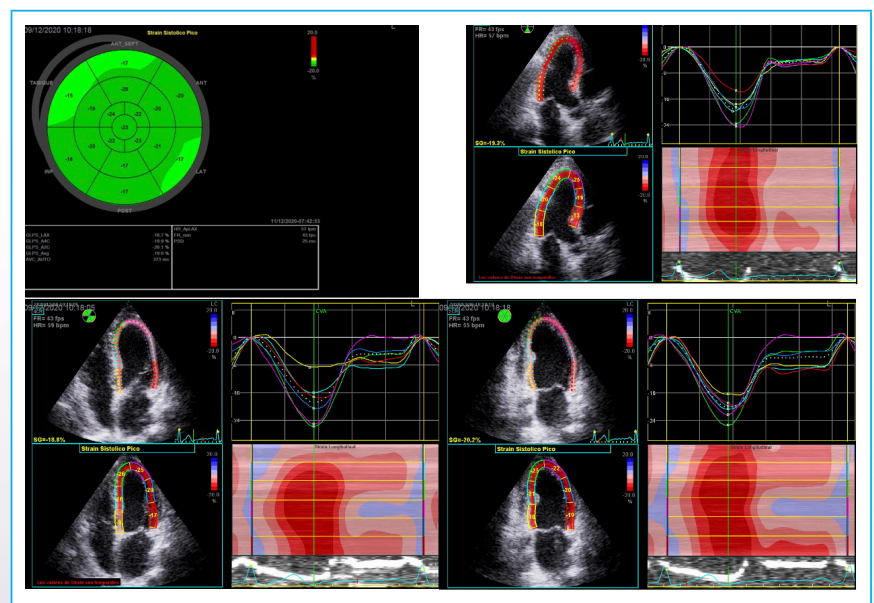

Figura 8. Strain longitudinal global a los 3 meses control.

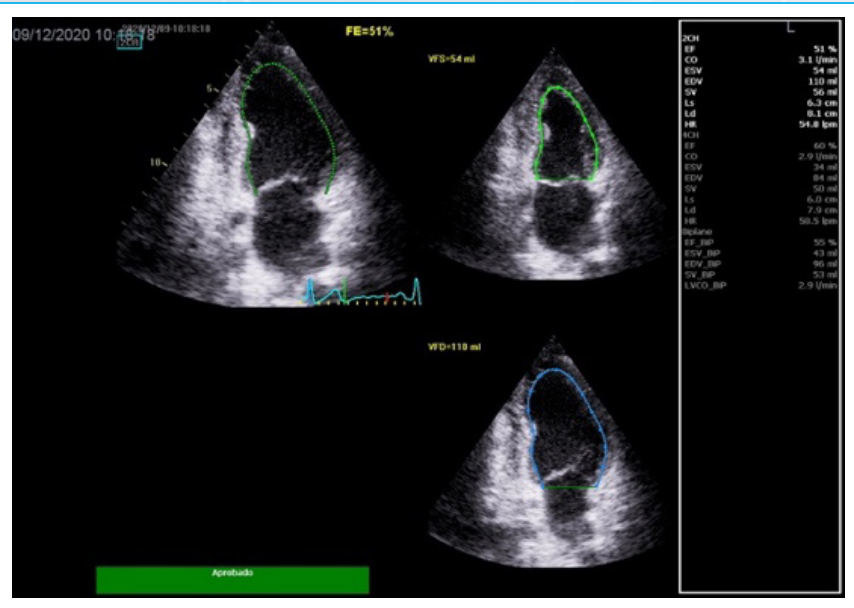

Figura 9. Ecocardiograma transtorácico a los tres meses de tratamiento anti falla cardíaca, que muestra recuperación de la fracción de eyección (automática).

\section{Discusión}

Los primeros casos de enfermedad por coronavirus (COVID 19) fueron reportados en diciembre 2019 en Wuhan, la capital de la provincia de Hubei, China ${ }^{1-7}$.
Las principales manifestaciones clínicas se originan por afectación del tracto respiratorio, incluida neumonía y síndrome de distrés respiratorio. Sin embargo, conforme la pandemia fue avanzando se identificó que se pueden presentar muchas manifestaciones extrapulmonares, incluidas las cardiovasculares, encontrándose incluso sin síntomas ni hallazgos clínicos de afección respiratoria

Existen muchos reportes de casos de pacientes con infección por SARS- CoV2 con daño o lesión miocárdica, sin embargo, no en todos los casos se ha esclarecido si dicha lesión miocárdica se debe directamente a la infección viral o si se origina como complicación indirecta de la enfermedad. Hay reporte de daño miocárdico como fenómeno tardío de la infección viral respiratoria

Si bien es cierto, las infecciones virales están ampliamente descritas como la causa más común de miocarditis, hasta el momento se sabe poco de la relación entre la lesión miocárdica y la infección por SARS- CoV-2 ${ }^{8}$.

Está descrito que puede haber presencia de daño microvascular debido al estado protrombótico que asocia la infección viral, y en algunos casos a posible presencia de coagulación intravascular diseminada. Además, la presencia de hipoxemia asociada a la enfermedad y el aumento en las demandas metabólicas pueden producir un desbalance entre el aporte y la demanda de oxígeno, provocando infarto tipo $2^{7}$. Sin embargo, el paciente no tuvo evidencia de ninguno de estos fenómenos.

Un aspecto muy importante es que la infección por SARS- CoV-2 puede desencadenar una respuesta inflamatoria excesiva, generando una tormenta de citoquinas, que por sí misma puede producir disfunción endotelial con edema miocárdico, sin estar directamente relacionado a infiltrados inflamatorios o presencia viral a nivel de los cardiomiocitos ${ }^{8}$. Dados los hallazgos clínicos y en los estudios de imagen, esta es la fisiopatología más probable del paciente en discusión.

También se ha propuesto que puede darse la entrada directa del virus a los miocitos cardíacos a través de receptores $A C E 2^{8}$, sin embargo, actualmente no hay evidencia histológica que lo corrobore.

La resonancia magnética cardíaca proporciona una caracterización morfológica y funcional del tejido, de forma no invasiva, brindando hallazgos altamente sugestivos de miocarditis ${ }^{8}$.

Asimismo, el papel de la biopsia endomiocárdica es incierto. Sala et al6, fueron los primeros en mostrar evidencia de presencia de inflamación miocárdica mediante biopsia en contexto de infección por COVID 19, documentando infiltrado linfocitario, edema intersticial importante, focos limitados de necrosis, y ausencia de fibrosis, lo que sugería un proceso inflamatorio agudo; in embargo, el análisis molecular mostró ausencia de genoma de SARS-CoV-2. En el reporte de una serie de autopsias se describió infección viral de células endoteliales en diferentes lechos vasculares, incluidas arterias coronarias, sin embargo, no se encontró signos de infiltrados linfocitarios miocárdicos. En dicho reporte se sugiere que la disfunción endotelial podría explicar la lesión miocárdica, especialmente en pacientes con factores de riesgo cardiovascular asociados ${ }^{6}$.

En otro reporte de caso, se documentó mediante biopsia presencia de partículas virales del tamaño típico del SARS-CoV-2 en miocardio de un paciente que asoció shock cardiogénico y que era positivo por este agente infeccioso, no obstante, los hallazgos histológicos mostraron pocos infiltrados inflamatorios y poca presencia de necrosis 8 .

En cuanto a la importancia clínica y la relevancia en el pronóstico del paciente, en un estudio realizado en New York, que incluyó cerca de 3000 indivi- 
duos hospitalizados y portadores de SARS- CoV-2, se evidenció que la lesión miocárdica es común en este grupo de pacientes, en donde la mayoría de los casos presentan manifestaciones clínicas leves, caracterizadas por elevación de troponinas. El riesgo de lesión miocárdica es mayor en los pacientes con patología cardíaca conocida previamente. En los casos que presentaron manifestaciones cardíacas más severas, se elevó significativamente el riesgo de mortalidad ${ }^{8}$

El tratamiento debe ser de soporte, donde se logre optimizar el tratamiento anti-falla cardíaca y se brinde un adecuado control de todas las comorbilidades asociadas.

\section{Conclusión}

Desde el inicio de la pandemia se han reportado muchos casos de miocarditis asociada al SARS- CoV-2, está claro que la infección por este virus comúnmente se asocia a elevación de troponinas y a daño miocárdico, lo cual se ha corroborado con hallazgos clínicos, ecocardiográficos y de resonancia magnética, confiriendo un peor pronóstico. La fisiopatología de la inflamación y daño miocárdico asociados a COVID 19 no está clara. En el caso presentado, los hallazgos clínicos e imagenológicos afirman la presencia de daño miocárdico reversible durante una infección activa por SARS- CoV-2 en vías de resolución.

\section{Ideas para recordar}

- La infección por SARS-COV-2 puede inducir daño miocárdico, el cual se manifiesta a través de síntomas y signos de falla cardíaca, elevación de biomarcardores cardíacos y alteración en la función cardíaca.

- Los estudios de imagen permiten identificar las alteraciones cardiacas iniciales y su evolución en el tiempo.

\section{Bibliografía}

1. Zhu N., Zhang D., Wang W. A novel coronavirus from patients with pneumonia in China, 2019. N Engl J Med. 2020;382:727-733.

2. Mahase E. Covid-19: death rate is $0.66 \%$ and increases with age, study estimates. BMJ. 2020;369:m1327.

3. Zeng J.-H., Liu Y.-X., Yuan J. First case of COVID-19 infection with fulminant myocarditis complication: case report and insights. Published online April 10 Infection. 2020 doi: 10.1007/s15010-020-01424-5

4. Inciardi R.M., Lupi L., Zaccone G. Cardiac involvement in a patient with coronavirus disease 2019 (COVID-19) JAMA Cardiol. 2020;5:819-824.

5. Kim I.-C., Kim J.Y., Kim H.A., Han S. COVID-19-related myocarditis in a 21-yearold female patient. Eur Heart J. 2020;41:1859.

6. Sala S., Peretto G., Gramegna M. Acute myocarditis presenting as a reverse Tako-Tsubo syndrome in a patient with SARS-CoV-2 respiratory infection. Eur Heart J. 2020;41:1861-1862

7. https://www.who.int/csr/don/12-january-2020-novel-coronavirus-china/en/

8. Inciardi RM, Lupi L, Zaccone $\mathrm{G}$, et al. Cardiac involvement in a patient with coronavirus disease 2019 (COVID-19). JAMA Cardiol. 2020;5(7):819-824. 